McGill/95-33

CERN-TH-95/137

hep-ph/9506285

June, 1995

\title{
Diffusion and Debye Screening Near Expanding Domain Walls
}

\author{
James M. Cline \\ McGill University, Montreal, PQ H3A 2T8, Canada, \\ and \\ Kimmo Kainulainen \\ CERN, CH-1211, Geneve 23, Switzerland.
}

\begin{abstract}
We study the effect of Debye screening of hypercharge when a net fermion number is reflected from a domain wall during a first order phase transition, which may be relevant for electroweak baryogenesis. We give a simple method for computing the effect of screening within the diffusion approximation, whose results are compatible with those of a more elaborate treatment based on the Boltzmann equation. Our formalism takes into account the differences in mobility of different particle species. We believe it is conceptually simpler than other accounts of screening that have appeared in this context. Somewhat surprisingly, we find that Debye screening can actually enhance electroweak baryogenesis by a modest factor $(\sim 2)$.
\end{abstract}


An important ingredient of the charge transport mechanism of electroweak baryogenesis [1, 2] is the diffusion of net left-handed fermion number into the false vacuum, after quarks and leptons reflect from expanding domain walls during the electroweak phase transition. This left-handed asymmetry is what drives sphalerons to produce a baryon asymmetry. It is desirable to understand how it propagates into the plasma because the more efficiently it does so, the longer it can drive baryon production before getting stopped by collisions and overtaken by the advancing wall. A number of papers have examined the diffusion problem from various angles [3]-[8].

In the early days of the charge transport proposal, there were some worries that the chiral asymmetry, because it carries hypercharge, might be prevented from penetrating deep into the plasma due to Debye screening, since hypercharge is an unbroken gauged charge [9]. Indeed this would be the case if there were only one species of fermion carrying both chirality and hypercharge. It was argued however that when there are many species, the hypercharge can be screened without implying that chirality is also screened [10, 11]. In fact reference [10] concluded that Debye screening has no effect whatever on the rate of baryon production (up to small effects due to the difference between Fermi-Dirac and Bose-Einstein statistics. In the present work we reexamine the effects of screening and show that in fact it can have some effect on the baryon violation rate, actually making it somewhat larger than if one had neglected screening. The reason for the discrepancy in conclusions is that we allow for the various species to have different diffusion coefficients, which leads to some particles being more efficient screeners than others. We show how to explicitly compute the effects of screening by incoporating the gauge forces responsible for it into the diffusion approximation. In this formalism one can derive rather elegantly the final values of particle asymmetries after screening and diffusion, starting from some initial asymmetries produced at the domain wall.

Our starting point, similarly to references [9] and [10], is the Boltzmann equation, including force terms for long range interactions. For each species of particle it is

$$
\left(\frac{\partial}{\partial t}+\vec{v} \cdot \frac{\partial}{\partial \vec{x}}+y_{i} \vec{E} \cdot \frac{\partial}{\partial \vec{x}}\right) f_{i}=\mathcal{C}_{i}
$$

where $y_{i}$ is the hypercharge of the $i$ th species, $\vec{E}$ is the field strength and $\mathcal{C}_{i}$ is a collision term. 
We take $f_{i}$ and $n_{i}$ below to refer to the net density of a given species, that is particle density minus antiparticle density. Integrating over momenta gives the usual continuity equation,

$$
\frac{\partial n_{i}}{\partial t}+\vec{\nabla} \cdot \overrightarrow{J_{i}}=0
$$

Although the screening force appears to have been lost, it reappears in the diffusion equation when we make a derivative expansion of the current in terms of the density, because one must also add an Ohm's law term to $\vec{J}$ :

$$
\overrightarrow{J_{i}}=-D_{i} \vec{\nabla} n_{i}+\sigma_{i} \vec{E}
$$

where $\sigma_{i}$ is the conductivity and $D_{i}$ the diffusion constant of the $i$ th species.

There is a simple relation between the conductivity and the diffusion coefficient. The latter can be expressed in terms of the mean interaction time and the mean squared velocity (between collisions) as $D=\overline{v^{2}} \tau / 3$, which for a relativistic particle is simply $\tau / 3$. The conductivity on the other hand is the induced current divided by $|\vec{E}|$, and the induced current is $n \bar{v}$, where $\bar{v}$ is the average velocity due to the biasing caused by the electric field. After a collision the electric field causes the particle to change its momentum by $\bar{p}=y_{i}|\vec{E}| \tau$ in the time before the next collision, and $\bar{v}=\bar{p} / p$. The result is that

$$
\sigma_{i}=3 n y_{i} D_{i} / p=y_{i} D_{i} T^{2} \begin{cases}0.33 & \text { bosons } \\ 0.21 & \text { fermions }\end{cases}
$$

Now we want to imagine the situation where a macroscopically large domain wall, moving with a velocity $v$ in the $z$ direction, is the source of the particle densities. Thus $\vec{\nabla} n$ becomes $\partial n / \partial z \equiv n^{\prime}$. Since we are interested in solutions of the form $n(z-v t)$, which would be static in the rest frame of the wall, we can replace $\partial n / \partial t$ with $-v n^{\prime}$. Furthermore the divergence of the electric field is given by Gauss's law, $\vec{\nabla} \cdot \vec{E}=\sum y_{i} n_{i}$. So

$$
v n_{i}^{\prime}+D_{i} n_{i}^{\prime \prime}-\sigma_{i} \sum_{j} y_{j} n_{j}=0
$$

or in vector notation on the space of species,

$$
v \vec{n}^{\prime}+\mathbf{D} \vec{n}^{\prime \prime}-(\vec{y} \cdot \vec{n}) \vec{\sigma}=0
$$


where $\mathbf{D}$ is the diagonal matrix with entries $D_{i}$. This equation can be readily solved using the Laplace transform,

$$
\vec{N}(s)=\int_{0}^{\infty} d z e^{-z s} \vec{n}(z)
$$

Making the definitions

$$
\begin{array}{rlrl}
\vec{n}_{0} & =\vec{n}(0) ; & \vec{n}_{0}^{\prime}=\vec{n}^{\prime}(0) ; \\
\mathbf{f} & =v s+\mathbf{D} s^{2} ; & \vec{\Sigma}=\mathbf{f}^{-1} \vec{\sigma} ; \\
\vec{g} & =s^{-1} \vec{n}_{0}+\mathbf{f}^{-1} \mathbf{D} \vec{n}_{0}^{\prime},
\end{array}
$$

equation (6) becomes $\vec{N}-(\vec{y} \cdot \vec{N}) \vec{\Sigma}=\vec{g}$. But by dotting $\vec{y}$ into this equation one can solve for $\vec{y} \cdot \vec{N}$ and therefore for $\vec{N}$,

$$
\vec{N}=\left(\mathbf{1}+\frac{\vec{\Sigma} \otimes \vec{y}}{1-\vec{\Sigma} \cdot \vec{y}}\right) \cdot \vec{g}
$$

The solution for $\vec{n}(z)$ can then be obtained from the inverse Laplace transform

$$
\vec{n}(z)=\oint \frac{d s}{2 \pi i} e^{z s} \vec{N}(s),
$$

where the contour is taken to encircle the left half-plane (including an infinitesimal vertical strip of the right half-plane). To evaluate $\vec{n}(z)$ we need to identify the residues of the poles of (9) contained within the contour. Furthermore the initial values $\vec{n}_{0}$ and $\vec{n}_{0}^{\prime}$ are related to each other by the physical requirement that the trivial constant solution does not exist and that the solution has the correct boundary condition $v n(0)=\vec{n}_{0}$. So one must eliminate $\vec{n}_{0}^{\prime}$ in favor of $\vec{n}_{0}$ to express the the solution in closed form.

Let us start with the simplest situation, in which there is only one kind of particle. Then

$$
N(s)=\left(1+\frac{\sigma y}{f-\sigma y}\right)\left(\frac{n_{0}}{s}+\frac{D n_{0}^{\prime}}{f}\right),
$$

which has poles at $s=0, s_{0}=-v / D$ and $s_{ \pm}=-v / 2 D \pm\left(v^{2}+4 D \sigma y\right)^{1 / 2} / 2 D$. But the residues at $s=0$ and $s=s_{0}$ vanish, and $s_{-}$is the only one remaining within the contour. The resulting solution in $z$-space is $n(z)=n_{0} \exp \left(s_{-} z\right)$. The effect of Debye screening is evident in this solution because the penetration depth is smaller than it would have been in the absence of the long-range force. In fact we can identify the Debye screening length as

$$
k_{D}^{-1}=(\sigma y / D)^{-1 / 2}
$$


When we take the limit of no screening, $\sigma y=0$, the solution of course reduces to that which has been previously found, $n_{0} \exp (-v z / D)$, showing that the penetration length gets longer as the wall gets slower, as one would expect from computing the time the wall takes to catch up with a diffusing particle.

Next consider the case where there are many particle species, but they all have the same diffusion coefficient, $D$. Again $\vec{N}(s)$ has poles at $s=0, s=s_{0}=-v / D$ and $s=s_{ \pm}$, but now $s_{ \pm}$is given by $s_{ \pm}=-v / 2 D \pm\left(v^{2}+4 D \vec{\sigma} \cdot \vec{y}\right)^{1 / 2} / 2 D$. In this case none of the residues automatically vanish; however the one at $s=0$ must be made to do so for physical consistency, since it would give a spatially constant solution. This gives the condition

$$
\mathbf{P} \cdot\left(\vec{n}_{0}+\frac{\mathbf{D}}{v} \vec{n}_{0}^{\prime}\right)=0,
$$

where $\mathbf{P}(\mathbf{Q})$ is a projection operator onto the subspace orthogonal (parallel) to hypercharge,

$$
\mathbf{P} \equiv \mathbf{1}-\mathbf{Q}=\left(\mathbf{1}-\frac{\vec{\sigma} \otimes \vec{y}}{\vec{\sigma} \cdot \vec{y}}\right) .
$$

Furthermore we get a relation between $\vec{n}_{0}$ and $\vec{n}_{0}^{\prime}$ from the requirement that $\vec{n}(z)$ indeed gives $\vec{n}_{0}$ at $z=0$,

$$
\mathrm{Q} \cdot \vec{n}_{0}^{\prime}=s_{-} \mathbf{Q} \cdot \vec{n}_{0}
$$

Thus the complete solution can be expressed in terms of $\vec{n}_{0}$,

$$
\vec{n}(z)=\left(e^{s_{0} z} \mathbf{P}+e^{s_{-} z} \mathbf{Q}\right) \cdot \vec{n}_{0},
$$

which has a quite simple geometric interpretation: there is only screening in the direction of particle species space proportional to hypercharge.

In the foregoing examples one could arrive at the solutions without recourse to the machinery of Laplace transforming, but in the general case where there are several diffusion coefficients, the solutions would be harder to guess. We assume that there are $M$ groups of particles classified by the values of their diffusion coefficients $D_{a}$ (for example different flavors of quarks have approximately equal diffusion constants because of their interactions with gluons). It is useful to define operators analogous to (14) that are nonzero only in the subspace of species with a given value $D_{a}$ :

$$
\mathbf{P}_{a} \equiv \mathbf{1}_{a}-\mathbf{Q}_{a}=\left(\mathbf{1}_{a}-\frac{\vec{\sigma}_{a} \otimes \vec{y}_{a}}{\vec{\sigma}_{a} \cdot \vec{y}_{a}}\right)
$$


where

$$
\begin{aligned}
& \mathbf{1}_{a} \equiv \lim _{\epsilon \rightarrow 0} i \epsilon\left(\mathbf{D}-D_{a}+i \epsilon\right)^{-1} \\
& \vec{\sigma}_{a}=\mathbf{1}_{a} \cdot \vec{\sigma} ; \quad \vec{y}_{a}=\mathbf{1}_{a} \cdot \vec{y} .
\end{aligned}
$$

There are $2 M$ physical solutions for the poles of $\vec{N}(s)$ which lie in the left-half plane. $M$ of these are obvious generalizations of the previous unscreened solution,

$$
s_{a}=-v / D_{a}
$$

The other $M$ physical poles, which we shall call $\bar{s}_{i}$, occur at the negative roots of an $(M+1)$ th order equation,

$$
\bar{s} \prod_{a}\left(\bar{s} D_{a}+v\right)-\sum_{a} \vec{\sigma}_{a} \cdot \vec{y}_{a} \prod_{b \neq a}\left(\bar{s} D_{b}+v\right)=0
$$

The complete solution can be written as

$$
\vec{n}(z)=\sum_{a=1}^{M} e^{s_{a} z} \mathbf{P}_{a} \cdot \vec{n}_{0}+\sum_{a, i, b=1}^{M} \vec{\sigma}_{a} \frac{M_{a i} e^{\bar{s}_{i} z}\left(M^{-1}\right)_{i b}}{\vec{\sigma}_{b} \cdot \vec{y}_{b}} \cdot \vec{n}_{0}
$$

where $M_{a i}$ is a square matrix with elements

$$
M_{a i}=\frac{1}{\bar{s}_{i}^{2} D_{a}+\bar{s}_{i} v}
$$

In practice it is not $\vec{n}_{0}$ but rather the fluxes $\vec{J}(0)$ at the boundary which are known at the outset. However it is straightforward to prove the intuitive result that

$$
\vec{J}(0)=v \vec{n}_{0}
$$

from eqs. (3) and (21) using the identities $D_{a} M_{a i} \bar{s}_{i}=\bar{s}_{i}^{-1}-v M_{a i}$, eq. (20), and the electric field $|\vec{E}(0)|=\int_{0}^{\infty} \vec{y} \cdot \vec{n} d z$.

It is trivial to verify that (21) satisfies the original differential equation; the nontrivial part is the determination of the roots $\bar{s}_{i}$. If we assume that $v^{2} / D \ll \vec{\sigma} \cdot \vec{y}$, which is the condition that the Debye screening length is much shorter than the usual diffusion length (and is always satisfied by the domain walls of the electroweak phase transition), it can be shown that two of the solutions to (20) are given by

$$
\pm\left(\vec{\sigma}_{a} \cdot \vec{y}_{a} / D_{a}\right)^{1 / 2}+O(v / D)
$$


of which the negative one is physical and gives rise to a single direction in the space of species which is screened. In the same regime of small velocities, the remaining $M-1$ solutions are approximately the roots of the truncated version of eq. (20) in which the first term (being of higher order in $v$ ) is omitted. This gives $M-1$ values of $\bar{s}_{i}$ which are all of order $v / D$ and therefore correspond to unscreened contributions.

To illustrate the full solution we will consider a few special cases of interest. First let us assume that there are just $M=2$ different diffusion coefficients, which could be useful for comparing the transport of quarks versus leptons, for example. Using eq. (四) and ignoring the difference between the bosons and fermions, we find the simple result

$$
\vec{n}(z)=\left(\sum_{a=1}^{2} e^{-v z / D_{a}} \mathbf{P}_{a}+\frac{e^{\bar{s} z}}{\vec{y} \cdot \vec{y}}\left(R_{2} \vec{y}_{1}-R_{1} \vec{y}_{2}\right) \otimes\left(\vec{y}_{1} / R_{1}-\vec{y}_{2} / R_{2}\right)\right) \cdot \vec{n}_{0},
$$

where

$$
\bar{s}=-\frac{v \vec{\sigma} \cdot \vec{y}}{D_{1} \vec{\sigma}_{2} \cdot \vec{y}_{2}+D_{2} \vec{\sigma}_{1} \cdot \overrightarrow{y_{1}}}=-\frac{v}{\vec{y} \cdot \vec{y}}\left(\frac{\vec{y}_{1} \cdot \vec{y}_{1}}{D_{2}}+\frac{\vec{y}_{2} \cdot \vec{y}_{2}}{D_{1}}\right)
$$

and

$$
R_{a}=\frac{\vec{y}_{a} \cdot \vec{y}_{a}}{\vec{y} \cdot \vec{y}}
$$

We have omitted the screened contribution here, which is concentrated much closer to the bubble wall than the unscreened parts. It can be checked that this result reduces to our previous one (16) in the limit that $D_{1} \rightarrow D_{2}$. It is not correct in the limit that one of the $D_{a}$ 's vanish, which corresponds to the decoupling of one of the particles, in the sense that it becomes immobile. This is because our assumption that $v^{2} / D \ll \vec{\sigma} \cdot \vec{y}$ is then violated, but since we expect this condition to be satisfied in nature even for quarks, which have the smallest diffusion length, eq. (26) is still the relevant one for considering the limit when one of the $D_{a}$ 's is much smaller than the other one.

The application motivating us to find this result was the case of differential reflection between chiral fermions and antifermions from the advancing domain wall as a means for producing the baryon asymmetry during the electroweak phase transition. In this mechanism, the net production of baryon number is proportional to the integrated density of $n_{B_{L}}+n_{L_{L}}$, a linear combination of the left-handed baryon and lepton number densities. We can write this as

$$
\Delta B=c \int_{0}^{\infty} d z \vec{u} \cdot \vec{n}(z)
$$


where $\vec{u}$ is a vector with components $u_{i}=1$ for every left-handed particle and zero for all others, and $c$ is some constant. It is easy to verify that $\vec{u} \cdot \vec{y}=0$, which stems from the fact that the baryon violation interaction giving rise to (28) is invariant under hypercharge gauge transformations. In the case of all equal diffusion coefficients $D_{i}$, this has the consequence that $\Delta B \propto \vec{u} \cdot \vec{n}_{0}$ because $\vec{u}$ is orthogonal to hypercharge,

$$
\vec{u} \cdot \mathbf{P} \cdot \vec{n}_{0}=\vec{u} \cdot \vec{n}_{0}
$$

This is the origin of the statement that hypercharge screening has no effect on the baryon asymmetry: we would have gotten the same answer if we had ignored screening altogether.

However when the differences between diffusivity of particle species are taken into account, it is no longer true that hypercharge screening has no effect because, for example,

$$
\vec{u} \cdot \mathbf{P}_{a} \cdot \vec{n}_{0} \neq \vec{u} \cdot \mathbf{1}_{a} \cdot \vec{n}_{0}
$$

because $\vec{u}$ is orthogonal to the full vector of hypercharges $\vec{y}$, but generally not to its projections $\vec{y}_{a}$ onto the space of species with diffusion coefficient $D_{a}$.

Let us next examine the case where there are $M=3$ distinct diffusion coefficients with the hierarchy $D_{1} \ll D_{2}<D_{3}$, which is the case for quarks and left-handed and right-handed leptons, respectively. Since $D_{1}$ is small we can expand in it. To zeroth order in $D_{1}$, the integrated densities in front of the wall are given by

$$
\int_{0}^{\infty} d z \vec{n}(z)=\left(\sum_{a=2}^{3} D_{a} \mathbf{P}_{a}+\frac{\left(R_{3} \vec{y}_{2}-R_{2} \vec{y}_{3}\right) \otimes\left(\vec{y}_{2} / R_{2}-\vec{y}_{3} / R_{3}\right)}{\vec{y}_{2} \cdot \vec{y}_{2} / D_{3}+\vec{y}_{3} \cdot \vec{y}_{3} / D_{2}}\right) \cdot \vec{n}_{0}
$$

which is just the same result as (25): we recover the results for $M=2$ when one of the particles becomes immobile $\left(D_{1} \rightarrow 0\right)$. The $O\left(D_{1}\right)$ corrections which involve the $\vec{y}_{1}$ direction either in the initial or the final density are

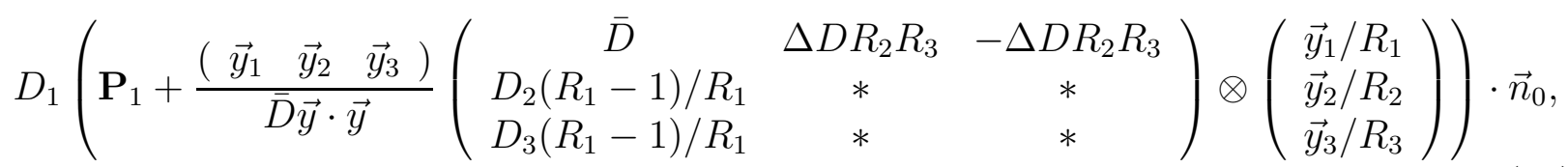

with

$$
\bar{D}=R_{2} D_{2}+R_{3} D_{3} ; \quad \Delta D=D_{3}-D_{2},
$$


and the $R_{a}$ 's defined as in eq. (27). From (32) we see that any particle densities induced by screening from an initial density of quarks (proportional to $\vec{y}_{1} \cdot \vec{n}_{0}$ if we identify quarks with the 1-direction), as well as any quark density induced from initial densities of leptons $\left(\vec{y}_{2,3} \cdot \vec{n}_{0}\right)$ by screening, propagates only with the diffusion coefficient $D_{1}$ of the quarks, which is much smaller than that of the leptons. Although this result may be intuitively obvious, the exact proportions by which these contributions are suppressed were not.

To obtain more concrete results, we need to specify the values of the hypercharges and the diffusion coefficients. The space of species consists of 18 left-handed quarks, 9 right-handed up-type quarks, 9 right-handed down-type quarks, 6 left-handed leptons, 3 right-handed leptons, and let us say 2 Higgs doublets, counting colors and flavors. We will take the Higgs bosons to have the same diffusion coefficient as the left-handed leptons. The hypercharge vector can thus be written as

$$
\begin{array}{rlrl}
\vec{y} & =\left(\vec{y}_{q} ; \vec{y}_{l} ; \vec{y}_{r}\right) & \\
\vec{y}_{q}=\left(18 \times \frac{1}{3} ; 9 \times \frac{4}{3} ; 9 \times-\frac{2}{3}\right) ; & \vec{y}_{q} \cdot \vec{y}_{q}=22 \\
\vec{y}_{l}=(6 \times-1 ; 2 \times-1) ; & \overrightarrow{y_{l}} \cdot \overrightarrow{y_{l}}=8 ; \\
\vec{y}_{r}=(3 \times-2) ; & \vec{y}_{r} \cdot \vec{y}_{r}=12 .
\end{array}
$$

Consider again the example with $M=2$, where we assume that right and left handed leptons have equal diffusion coefficients (so that $\vec{y}_{\text {lepton }}=\vec{y}_{l} \oplus \vec{y}_{r}$ ). Then assuming that the inital flux consisted only of left handed bottom quarks with total density $n_{q}$ and right handed bottom quarks with density $-n_{q}$ and similarly for leptons with density $\pm n_{l}$, we would find

$$
\Delta B=c v^{-1}\left(n_{q} D_{q}+n_{l} D_{l}\right)-3 c v^{-1} \frac{D_{l}-D_{q}}{11 D_{q}+10 D_{l}}\left(D_{q} n_{q}-D_{l} n_{l}\right) .
$$

This result implies an enhancement in the flux due to the hypercharge screening by about 30 per cent. It also illustrates the fact that regardless of screening, the particles with the largest diffusion coefficient are still the most efficacious for producing baryon number.

The most interesting physical application is the situation where the initial state at the wall consisted only of left-handed tau leptons with density $n_{\tau}$ and an equal and opposite density $-n_{\tau}$ of right-handed tau leptons (since the process of reflection of fermions from the 
wall conserves lepton number) [2, [2]. Neglecting the quark contribution entirely, we then find that

$$
\Delta B=c v^{-1} \frac{D_{l}}{4}\left(1+\frac{7}{1+\left(2 D_{l}\right) /\left(3 D_{r}\right)}\right) \simeq \frac{7}{4} c v^{-1} D_{l},
$$

using that the ratio of diffusion constants of left- and right-handed leptons is approximately $D_{l} / D_{r} \cong 1 / 4$. The naive answer ignoring screening is $\Delta B=c D_{l} / v$. We thus see again the curious effect that screening increases the net amount of propagation of left-handed lepton number into the plasma.

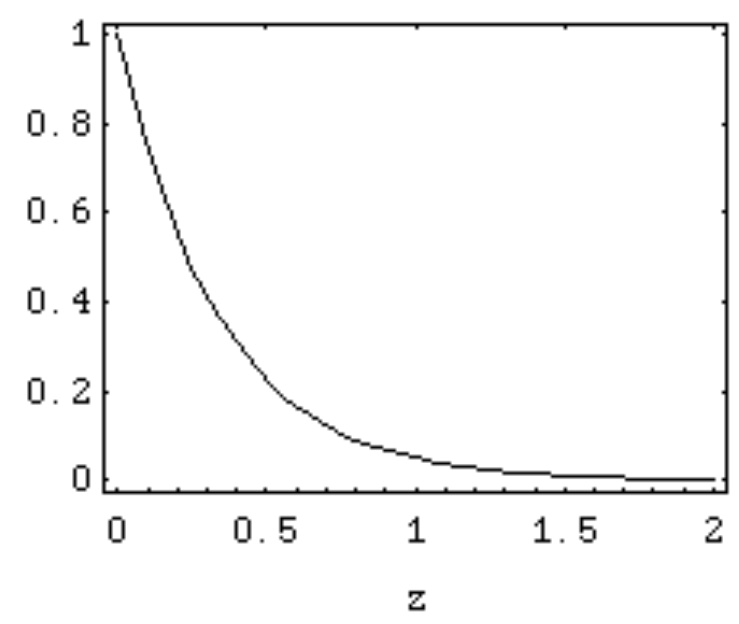

Figure $1 \mathrm{a}$

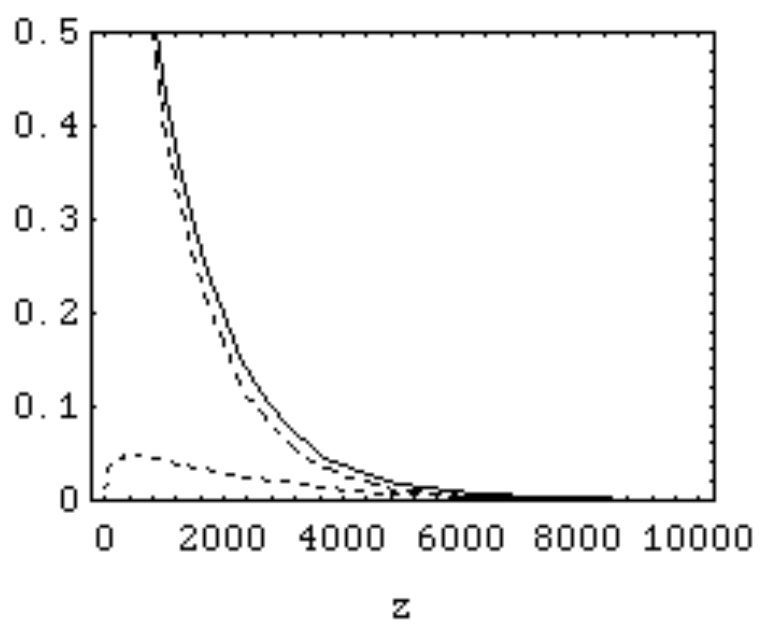

Figure 1b

Figure 1. Figure 1a shows the hypercharge density in front of the wall. Figure 1b shows the spatial distribution of the asymmetries of the left handed fermions. The solid line corresponds to the numerical solution of equation (21) for the $\tau_{L}$-asymmetry in the presence of screening and the short dash - long dash one to the $\tau_{L}$-asymmetry in the absence of screening. The dashed line represents the $e_{L}, \mu_{L}$ and $\nu_{i L}$-asymmetries $(i=e, \mu, \tau)$, which would be zero in the absence of the hypercharge screening. Distance is in units of $T^{-1}$.

We show some results from the full numerical solution of the equation (21) corresponding to the above case of initial tau fluxes only, and with $D_{q}=6 / T, D_{l}=110 / T$ and $D_{r}=4 D_{l}$. Figure 1a shows the hypercharge density in front of the wall. The width of the distribution is seen to correctly correspond to the inverse of the eigenvalue (24). Figure 1b shows the distributions of the asymmetries of left handed leptons. Note in particular the distributions of the $e_{L}, \mu_{L}$ and neutrino-asymmetries that the hypercharge screening pulls out from the 
plasma. It is the appearance of these new leptonic asymmetries, along with the fact that the $\tau_{L}$ asymmetry is only slightly reduced by the screening, that explains the enhancement in the baryon production found above in the analytic formula (36). The precise numerical result for the final baryonic asymmetry is close to the analytic estimate: $\Delta B \simeq 1.69 \mathrm{cv}{ }^{-1} D_{l}$.

Let us finally caution the reader that there may be problems with the fundamental assumptions in the diffusion approach; Fick's law implicitly assumes that the reflected asymmetry current has a near-thermal distribution, whereas the initial flux emanating from the wall is rather strongly concentrated at low momenta [1, 3, 12]. It remains to be seen how much this will affect the above results of the diffusion computation.

In conclusion, we have studied the diffusion of a reflected fermion current from the domain wall during a first order phase transition, which is relevant for the charge transport mechanism of electroweak baryogenesis. In our treatment we included the effect of Debye screening of hypercharge and accounted for the differences in mobility of different particle species. Our main result is that in the case where the baryogenesis is driven by the flux of reflected tau leptons, Debye screening actually enhances electroweak baryogenesis by a modest factor $\sim 1.7$.

\section{References}

[1] A.G. Cohen, D.B. Kaplan and A.E. Nelson, Nucl. Phys. B349, 727 (1991); Nucl. Phys. B373, 453 (1992).

[2] M. Joyce, T. Prokopec and N. Turok, Phys. Lett. B338, 269 (1994).

[3] J.M. Cline, Phys. Lett. B338, 263 (1994).

[4] A.G. Cohen, D.B. Kaplan and A.E. Nelson, Phys. Lett. B336, 41 (1994).

[5] M. Joyce, T. Prokopec and N. Turok, preprint PUPT-1496, hep-ph/9410282.

[6] J. Maalampi, J. Sirkka and I. Vilja, Phys. Lett. B337, 122 (1994).

[7] D. Comelli, M. Pietroni and A. Riotto, preprint DFPD 94/TH/39, hep-ph/9406369.

[8] G. Farrar and J.W. McIntosh, Jr., preprint RU-94-59, hep-ph/9412270 (1994). 
[9] S. Yu. Khlebnikov, Phys. Lett. B300, 376 (1993).

[10] A.G. Cohen, D.B. Kaplan and A.E. Nelson, Phys. Lett. B294, 57 (1992).

[11] M. Joyce, T. Prokopec and N. Turok, preprint PUPT-1495, hep-ph/9410281.

[12] J.M. Cline, K. Kainulainen and A.P. Vischer, preprint McGill-95/16, hep-ph/9506284. 